\title{
Characteristic Features of Stone-Wales Defects in Single-Walled Carbon Nanotube; Adsorption, Dispersion, and Field Emission
}

\author{
Seungkwang Roh, ${ }^{1}$ Jongtaek Lee, ${ }^{2}$ Mira Jang, ${ }^{2}$ Mingyeong Shin, ${ }^{2}$ Juone Ahn, ${ }^{2}$ Taehee Park, ${ }^{2}$ \\ and Whikun $\mathbf{Y i}^{2}$ \\ ${ }^{1}$ Paju LCD Industrial Complex, Paju-city, Gyeonggi-do 413-779, Republic of Korea \\ ${ }^{2}$ Department of Chemistry, Hanyang University, Haengdang-dong 17, Seoul 133-791, Republic of Korea
}

Correspondence should be addressed to Whikun Yi, wkyi@hanyang.ac.kr

Received 31 May 2010; Accepted 29 August 2010

Academic Editor: Rakesh Joshi

Copyright ( $) 2010$ Seungkwang Roh et al. This is an open access article distributed under the Creative Commons Attribution License, which permits unrestricted use, distribution, and reproduction in any medium, provided the original work is properly cited.

\begin{abstract}
Adsorption behaviors of dodecanethiol $\left(\mathrm{C}_{12} \mathrm{H}_{25} \mathrm{SH}\right)$ molecules are investigated on the surface of single-walled carbon nanotubes (SWCNTs) with vibrational and X-ray photoelectron spectrometers. The active adsorption sites are proved as Stone-Wales (SW) defects (5-7 ring defects). The SW defect-removed SWCNTs formed by reacting nanotubes with allyl acrylate molecules are compared with pristine SWCNTs in dispersion and field emission. The former shows higher dispersion and field emission than the latter.
\end{abstract}

\section{Introduction}

Carbon nanotubes (CNTs) have a lot of defects such as, vacancies, metastable atoms, pentagons, heptagons, StoneWales (SW or a pair of 5-7 rings) defects, discontinuities of walls, and heterogeneous atoms [1-5]. According to a scanning tunneling microscopy (STM) observation, about $10 \%$ of the samples were found to exhibit stable defect features under extended scanning [6]. SW defects are stable and commonly present in CNTs and are believed to play key roles in the mechanical [7], electronic [8], and chemical [9] properties of CNTs.

In the studies of the interaction of CNTs with organic compounds, amines have attracted special attention [1014]. Among these, the most extensively explored is the formation of amide derivatives between carboxylic groups on oxidized CNT tips and long-chain amines. Basiuk et al. [15] reported that infrared (IR) spectra of oxidized singlewalled CNTs (SWCNTs) treated with amines under different conditions could not correspond to amide derivatives on SWNCT tips, because the very low concentration of the terminal groups relative to the whole sample mass resulted in a negligible contribution to the IR spectra. The bands detectable in the case of long-chain amines were thought to correspond to amine molecules physisorbed because of strong hydrophobic interactions of their hydrocarbon chains with SWCNT walls. Our previous studies [16] investigated the adsorption behaviors of alkanethiol molecules on the surface of SWCNTs with vibrational spectrophotometer. We reported that alkanethiol molecules adsorbed strongly on the SWCNT surface and suggested that the active activation sites in the nanotube surface might be SW defects. According to the theoretical calculation by Zhou and Shi [17], it was found that the SW defects of the tube wall could reduce cohesive energies for some foreign atoms such as, H, C, N, O, F, and P. In other words, the heptagon rings generated during SWCNT synthesis can be good candidates for an adsorption of foreign molecules. More recently, first-principle calculations were performed to investigate the dependence of alkanethiol's interaction with SWCNTs on nanotube's type, curvature, and chirality [18]. They reported that the affinities of ethanethiol molecules for the semiconducting and metallic SWCNTs were rather close, about $10 \%$ stronger in the former case, and the binding energy of alkanethiol was increased for adsorption on large diameter CNTs.

In this study, we investigate the effect of SW defects for an adsorption of dodecanethiol (DT, $\left.\mathrm{C}_{12} \mathrm{H}_{25} \mathrm{SH}\right)$ molecules onto SWCNT surface to get more advanced evidence on 
active sites compared with our previous results. Moreover, after removing (or changing) SW defects from nanotube surface, the degree of dispersion and field emission (FE) of SWCNTs, respectively, was measured and compared with that of untreated pristine SWCNTs.

\section{Experimental}

SWCNTs and MWCNTs produced by arc-discharge process and thermal chemical vapor deposition (CVD) technique, respectively, were used for an adsorption of dodecanethiol (DT). Both nanotubes were oxidized thermally to remove carbon-containing materials, and subsequently sonificated in $\mathrm{HNO}_{3}$. Both CNTs were dispersed in ethanol by an ultrasonification for $8 \mathrm{~h}$, sprayed on a glass, and then dried. The CNTs were immersed in the DT-containing ethanol solution for $1 \mathrm{~min}$, and successively washed out several times with ethanol to remove physisorbed DT molecules from the surface of nanotubes. Adsorbed DT molecules were characterized by infrared (IR) and X-ray photoelectron spectrometer (XPS).

The removal (change) of 5-7 ring defects was carried out by reacting SWCNTs with methyl acrylate or octyl acrylate at $120^{\circ} \mathrm{C}$ (Diels-Alder reaction). Ultraviolet- (UV-) visible spectrometer was chosen to measure the degree of dispersion of SWCNTs in solvent. The field emission (FE) properties were measured using a two-parallel plate, that is, diode type, in a vacuum pressure of $\sim 1.0 \times 10^{-6}$ Torr at room temperature. Indium tin oxide (ITO) was used for two electrodes and the distance between them was kept at $300 \mu \mathrm{m}$.

\section{Results and Discussion}

Figure 1 shows the vibrational spectra of pristine SWCNTs, methyl acrylate-treated defect-removed SWCNTs, and MWCNTs after an adsorption of DT molecules. In case of pristine SWCNTs, the spectrum contains strong two bands at 2856 and $2927 \mathrm{~cm}^{-1}$ which is assigned to symmetric and asymmetric stretching of $-\mathrm{CH}_{2}$ - of adsorbed DT molecules, respectively. The shoulder around $2960 \mathrm{~cm}^{-1}$ is assigned to the asymmetrical stretching of $\mathrm{CH}_{3}$ [19-21]. Our adsorption experiments were performed in liquid phase and through a consecutive washing condition. Therefore, our case would allow only the strong adsorption between DTs and SWCNTs, probably chemisorption.

The MWCNTs show no vibrational peaks of DTs, indicating that DTs are hardly adsorbed on the surface of MWCNTs. To understand the interactions between DT molecules and nanotube surface, we observed XPS spectra in the sulfur (2p) region of DT molecules. Figures 2(a) and 2(b) show an Mg K $\alpha$-induced XPS spectrum of sulfur (2p) core levels of DT molecules on SWCNT and MWCNT surface, respectively. In general, the $S(2 \mathrm{p})$ spectra are composed of $2 \mathrm{p}_{3 / 2}$ and $2 \mathrm{p}_{1 / 2}$ peaks with an intensity of $2: 1$, as theoretically determined from the spin-orbit splitting effect [22-26]. Figure 2(a) shows two peaks at $162.8 \mathrm{eV}\left(\mathrm{S}_{2} \mathrm{p}_{3 / 2}\right)$ and $164.4 \mathrm{eV}\left(\mathrm{S}_{2} \mathrm{p}_{1 / 2}\right)$. Deconvolution of the sulfur $(2 \mathrm{p})$

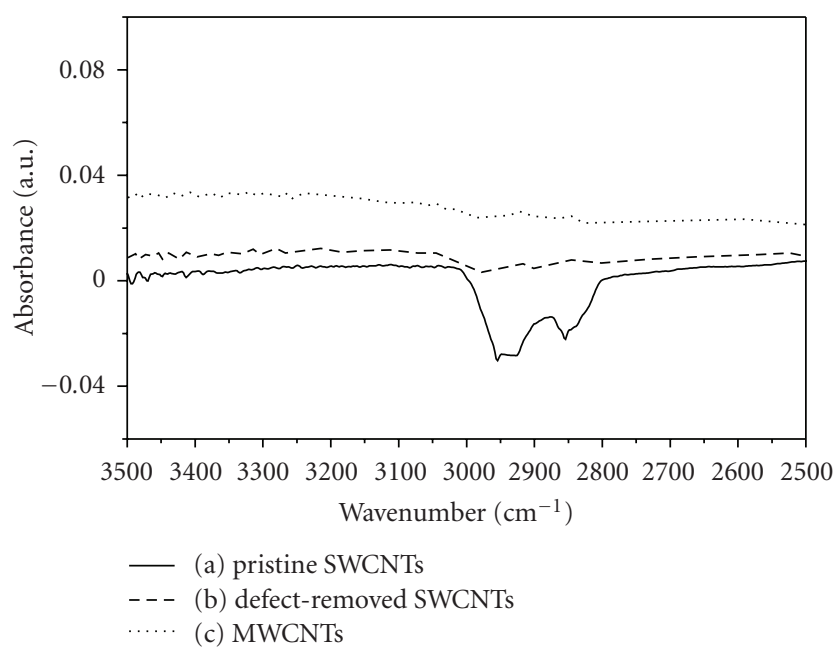

FIGURE 1: IR spectra after an adsorption of alkanethiol (DT) molecules for (a) pristine SWCNTs, (b) methyl acrylate-treated (defect-removed) SWCNTs, and (c) MWCNTs.

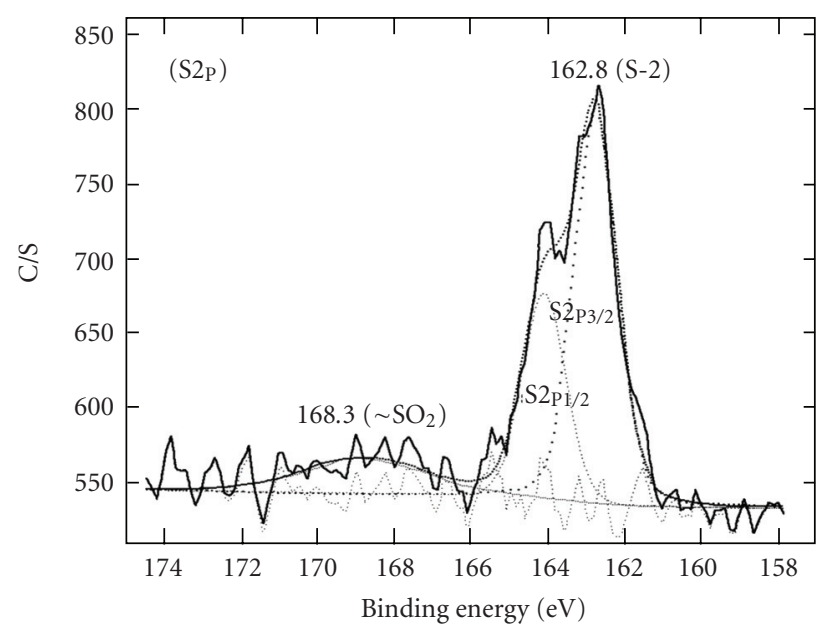

(a)

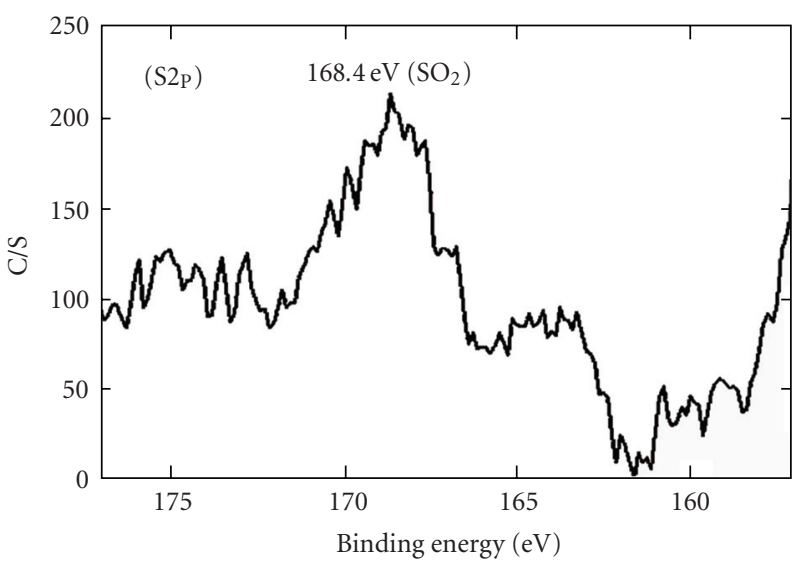

(b)

FIGURE 2: S2p XPS spectra of (a) DT-adsorbed SWCNTs and (b) DT-adsorbed MWCNTs. 
emission line shape reveals the characteristic intensity ratio $2: 1$ with the peak separation of $1.2 \mathrm{eV}$. The binding energy of this state suggests the presence of a C-S bond, consistent with chemisorbed thiolate $[23,24,26]$. On the other hand, we cannot see $\mathrm{S} 2 \mathrm{p}_{3 / 2}$ and $\mathrm{S} 2 \mathrm{p}_{1 / 2}$ peaks on MWCNT surface (Figure 2(b)), which strongly suggests that there is no formation of thiolate species. In other words, DT molecules are not adsorbed on the MWCNT surface. The broad peak around $168.4 \mathrm{eV}$ is believed due to sulfonate (or sulfoxide) species.

For the methyl acrylate-treated SWCNTs (Figure 1(b)), the two peaks at 2856 and $2927 \mathrm{~cm}^{-1}$ reduce drastically or almost disappear, which is the same result in our previous report [16]. We suggested that the active adsorption sites in SWCNTs might be SW defects for an adsorption of alkanethiols, thus DTs could not be adsorbed on the surface of SWCNTs after removal of SW defects artificially by reacting methyl acrylate $\left(\mathrm{CH}=\mathrm{CH}-\mathrm{C}=\mathrm{O}\left(\mathrm{OCH}_{3}\right)\right)$ with SWCNTs via Diels-Alder reaction in Scheme 1.

When we treated with methyl acrylate to remove SW defects, the reaction products were not evident since any characteristic vibrational peaks of the products were not found in the IR spectrum. It would be attributed by the small amount of the product sample.

We used octyl acrylate $\left(\mathrm{CH}=\mathrm{CH}-\mathrm{C}=\mathrm{O}\left(\mathrm{OC}_{8} \mathrm{H}_{17}\right)\right)$ instead of methyl acrylate to treat SWCNT surface. Octyl acrylate molecule has longer chain length and more carbon atoms inside than methyl acrylate, thus it is expected that vibrational spectrum due to long chain carbon atoms would be shown if octyl acrylate reacts with SWCNT surface through chemical reaction. Figure 3 represents the IR spectrum of octyl acrylate-treated SWCNTs. Inset shows IR spectrum of neat liquid sample of octyl acrylate molecules for comparison. We can see clearly the peaks at 2856, 2927, and $1725 \mathrm{~cm}^{-1}$ corresponding to symmetric and asymmetric stretching of $-\mathrm{CH}_{2-}$, and conjugate $\mathrm{C}=\mathrm{O}$ stretching of octyl acrylate, respectively. The intensity of conjugated $\mathrm{C}=\mathrm{C}$ stretching mode at 1639 and $1618 \mathrm{~cm}^{-1}$ is reduced largely compared with the neat sample in inset. IR spectrum results strongly suggest that the 5-7 defects on SWCNTs react with octyl acrylate molecules via Diels-Alder reaction in Scheme 1. SWCNT tips are not considered here since terminated carboxyl $(-\mathrm{COOH})$ or hydroxyl $(-\mathrm{OH})$ groups hardly react with methyl acrylate.

Vibrational intensities of DT molecules are negligible on the surface of MWCNTs as shown in Figure 1(c). As elucidated in XPS results (Figure 2), DT molecules cannot adsorb on MWCNT surface chemically. This implies that the MWCNT surface has a relatively small number of 57 ring defects. According to theoretical examination [17], the energy barrier for bond rotation to form 5-7 defects from hexagonal rings is smaller in the tubes than in planar graphene because of the additional tubular strain. The above statements imply that an SWCNT has an advantage over an MWCNT to form 5-7 ring defects since the energy barrier for bond rotation in the SWCNT is smaller than in the MWCNT due to higher tubular strain. Consequently, the SWCNT has more chance to make 5-7 defects during synthesis than the MWCNT.

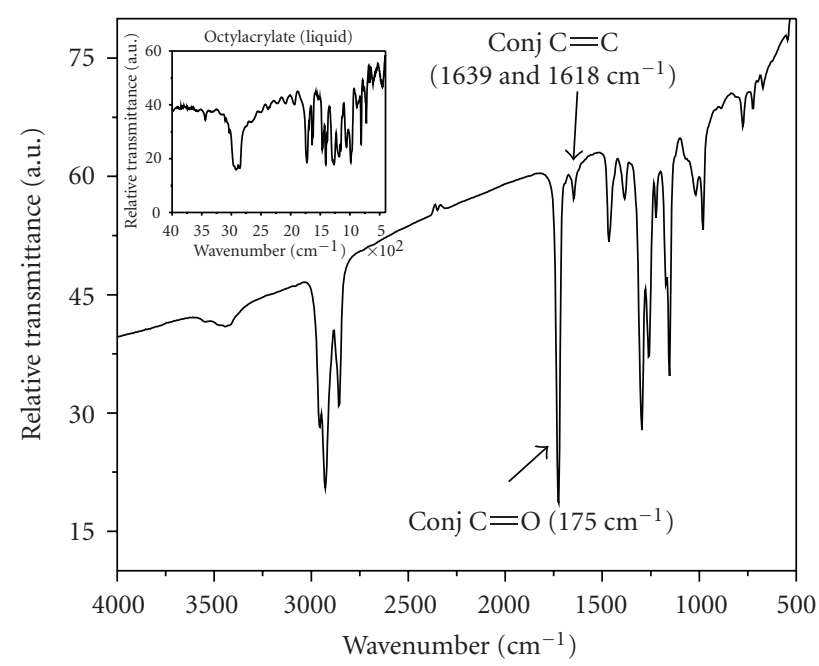

FIGURE 3: IR spectrum of octyl acrylate-treated SWCNTs. Inset represents vibrational spectrum of neat octyl acrylate.

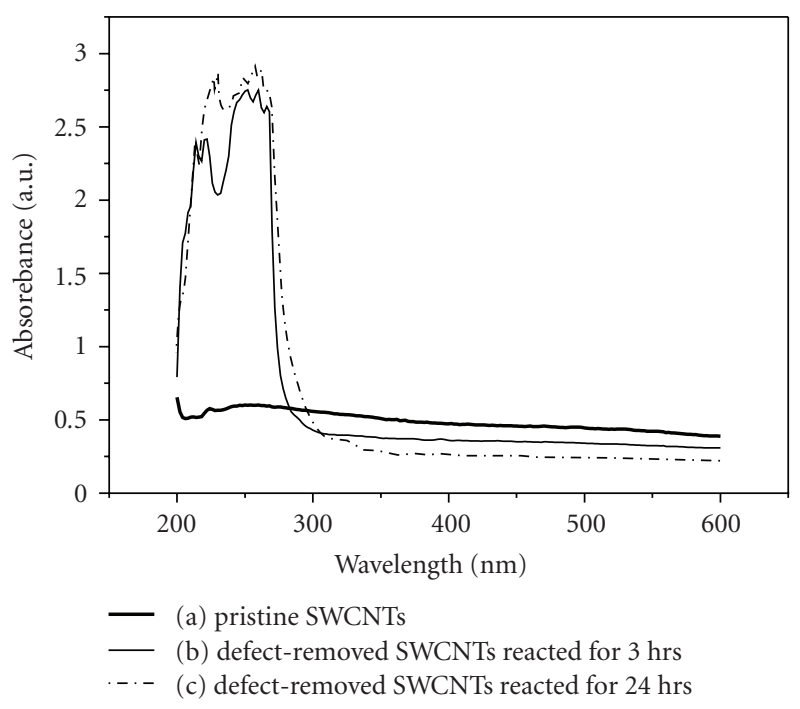

FIgURE 4: UV spectra of pristine SWCNTs (a), methyl acrylatetreated SWCNTs reacted for $3 \mathrm{hrs}$ (b) and for $24 \mathrm{hrs} \mathrm{(c).}$

Figure 4 shows UV-visible absorption spectra of pristine SWCNTs, methyl acrylate-treated SWCNTs (defect-removed SWCNTs) reacted for $3 \mathrm{hrs}$ and $24 \mathrm{hrs}$ in ethanol, respectively. More intense absorption from the spectra implies more stable homogeneous dispersion of SWCNTs in solvent $[12,14,27]$. Therefore, it can be said that the methyl acrylatetreated SWCNTs are dispersed more well rather than the pristine SWCNTs.

SWCNTs always form aggregates because of very strong van der Waals interaction between them. Keeping stable dispersion of SWCNTs in solvent is significant, and also a perquisite for application as additives for reinforcement of composite materials. SWCNTs were solubilized in water with the aid of surfactant, purified and length-selected [28-30], but removing the surfactant afterwords was problematic. 


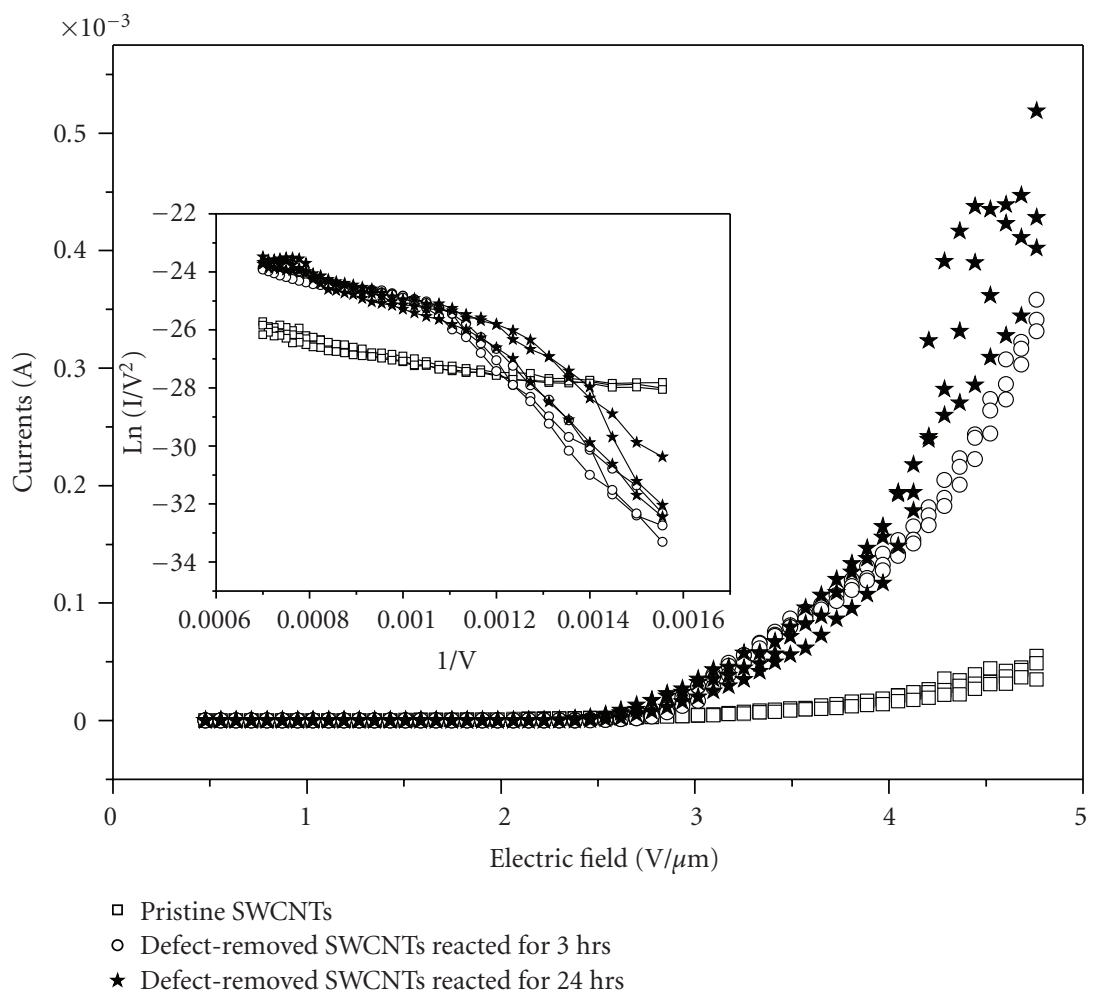

(a)

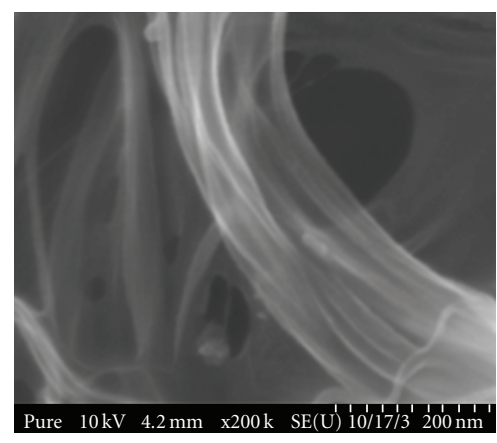

Pristine SWCNTs

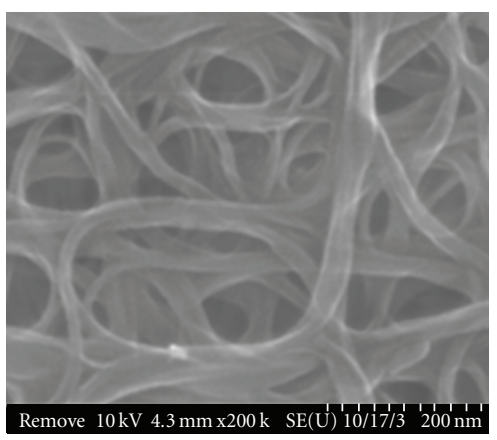

Methylacrylate-treated SWCNTs

(b)

FIGURE 5: Field emission I-V curves (a) and SEM pictures (b) of pristine SWCNTs, methyl acrylate-treated SWCNTs reacted for 3 hrs and 24 hrs, respectively.
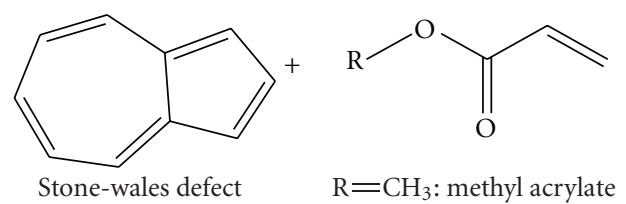

$120^{\circ} \mathrm{C}, 1$ day $\underset{\text { Diels-alder reaction }}{\stackrel{\text { under argon gas }}{\longrightarrow}}$

$$
\begin{aligned}
\mathrm{R} & =\mathrm{CH}_{3}: \text { methyl acrylate } \\
& =\mathrm{C}_{8} \mathrm{H}_{17} \text { : octyl acrylate }
\end{aligned}
$$

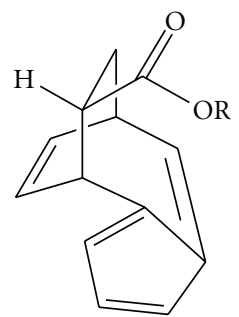

Scheme 1: Diels-Alder reaction to remove (change) SW defects (5-7 ring defects) on the surface SWCNTs. 
SWCNTs were also solubilized by functionalizing the endcaps with long aliphatic amines. The above approaches apparently work well, but have the disadvantage that the most convenient chemical handles further modification, and the acid-treated end-caps are tied-up by the solubilization functionality. Our method to remove ring defects is useful since it works well as shown in Figure 4. The interaction between SWCNTs is thought as a hydrophobic force. More stable homogeneous dispersion would be the results that the protruded acrylate molecules attached on the SWCNT surface can make SWCNTs to be exfoliated from the ropes.

We measured field emission (FE) current density of pristine SWCNTs, methyl acrylate-treated SWCNTs for 3 hrs and 24 hrs, respectively. Figure 5(a) shows that methyl acrylatetreated SWCNTs have higher FE current density than pristine SWCNTs, and also FE current behavior of methyl acrylatetreated SWCNTs is similar for both 3- and 24-hour-reacted sample in solvent. The turn-on field of each sample, $\mathrm{V}_{\mathrm{T}-\mathrm{O}}$, which is defined as the macroscopic field to produce a current density of $10 \mu \mathrm{A} / \mathrm{cm}^{2}$, was obtained from the logarithmic plot of Figure 5(a). The turn-on field decreased from $3.57 \mathrm{~V} / \mu \mathrm{m}$ (pristine SWCNTs) to 2.8 and $2.73 \mathrm{~V} / \mu \mathrm{m}$ for 3 hrs and 24 hrs, respectively. The maximum emission current density gradually increased and reached $0.50 \mathrm{~mA} / \mathrm{cm}^{2}$ for the 24-hour-reacted defect-removed SWCNTs, about ten times higher than pristine SWCNTs $\left(0.05 \mathrm{~mA} / \mathrm{cm}^{2}\right)$ at an electric field of $4.7 \mathrm{~V} / \mu \mathrm{m}$. The same explanations can be adopted for the enhanced FE current density, that is, the protruded acrylate molecules on the SWCNT surface can make SWCNTs exfoliate from the ropes as shown in Figure 5(b). The well separated SWCNTs reduce the screening effect [31], which leads to an emission decrease caused by neighboring tips when the distance between tips is too small.

\section{Conclusion}

In summary, Thiol-containing DT molecules can be adsorbed on the surface of SWCNTs, not MWCNTs, and the active adsorption sites are proved as 5-7 ring defects of the nanotube surface which can be removed (changed) by reacting with allyl acrylate molecules via Diels-Alder reaction. The defect-removed SWNCTs show higher degree of dispersion in solvent and enhanced FE currents compared with pristine SWCNTs.

\section{Acknowledgment}

The authors gratefully acknowledge the Korean Science and Engineering Foundation (KRF-2009-007-2914 and KRF2010-001-6233).

\section{References}

[1] F. Banhart, "Irradiation effects in carbon nanostructures," Reports on Progress in Physics, vol. 62, no. 8, pp. 1181-1221, 1999.

[2] S. Lijima, T. Ichihashi, and Y. Ando, "Pentagons, heptagons and negative curvature in graphite microtubule growth," Nature, vol. 356, no. 6372, pp. 776-778, 1992.
[3] O. Zhou, R. M. Fleming, D. W. Murphy et al., "Defects in carbon nanostructures," Science, vol. 263, no. 5154, pp. 17441747, 1994.

[4] J.-C. Charlier, "Defects in carbon nanotubes," Accounts of Chemical Research, vol. 35, no. 12, pp. 1063-1069, 2002.

[5] E. Saether, "Transverse mechanical properties of carbon nanotube crystals_-part II: sensitivity to lattice distortions," Composites Science and Technology, vol. 63, no. 11, pp. 15511559, 2003.

[6] M. Ouyang, J.-L. Huang, C. L. Cheung, and C. M. Lieber, "Atomically resolved single-walled carbon nanotube intramolecular junctions," Science, vol. 291, no. 5501, pp. 97100, 2001.

[7] K. M. Liew, X. Q. He, and C. H. Wong, "On the study of elastic and plastic properties of multi-walled carbon nanotubes under axial tension using molecular dynamics simulation," Acta Materialia, vol. 52, no. 9, pp. 2521-2527, 2004.

[8] E. J. Duplock, M. Scheffler, and P. J. D. Lindan, "Hallmark of perfect graphene," Physical Review Letters, vol. 92, no. 22, Article ID 225502, 1 pages, 2004.

[9] S. Picozzi, S. Santucci, L. Lozzi, L. Valentini, and B. Delley, "Ozone adsorption on carbon nanotubes: the role of StoneWales defects," Journal of Chemical Physics, vol. 120, no. 15, pp. 7147-7152, 2004.

[10] S. S. Wong, E. Joselevich, A. T. Woolley, C. L. Cheung, and C. M. Lieber, "Covalently functionalized nanotubes as nanometresized probes in chemistry and biology," Nature, vol. 394, no. 6688, pp. 52-55, 1998.

[11] S. S. Wong, A. T. Woolley, E. Joselevich, C. LI. C. , and C. M. Lieber, "Covalently-functionalized single-walled carbon nanotube probe tips for chemical force microscopy," Journal of the American Chemical Society, vol. 120, no. 33, pp. 85578558, 1998.

[12] J. Chen, M. A. Hamon, H. Hu et al., "Solution properties of single-walled carbon nanotubes," Science, vol. 282, no. 5386, pp. 95-98, 1998.

[13] M. A. Hamon, J. Chen, H. Hu et al., "Dissolution of singlewalled carbon nanotubes," Advanced Materials, vol. 11, no. 10, pp. 834-840, 1999.

[14] K. D. Ausman, R. Piner, O. Lourie, R. S. Ruoff, and M. Korobov, "Organic solvent dispersions of single-walled carbon nanotubes: toward solutions of pristine nanotubes," Journal of Physical Chemistry B, vol. 104, no. 38, pp. 8911-8915, 2000.

[15] E. V. Basiuk, V. A. Basiuk, J.-G. Banuelos et al., "Interaction of oxidized single-walled carbon nanotubes with vaporous aliphatic amines," Journal of Physical Chemistry B, vol. 106, no. 7, pp. 1588-1597, 2002.

[16] S. Roh, J. Oh, Y. Choi et al., "Adsorption of alkanethiol molecules onto carbon nanotube surface," Journal of Vacuum Science and Technology B, vol. 22, no. 3, pp. 1411-1415, 2004.

[17] L. G. Zhou and S. Q. Shi, "Adsorption of foreign atoms on Stone-Wales defects in carbon nanotube," Carbon, vol. 41, no. 3, pp. 613-615, 2003.

[18] J. Shakhs Emampour, M. D. Ganji, S. Mahmoudi, M. M. Taghavi, and M. Shokry, "Effect of curvature and chirality for alkanethiols interaction with single-walled carbon nanotubes: ab initio investigation," Iranian Journal of Organic Chemistry, vol. 4, pp. 256-260, 2009.

[19] M. D. Porter, T. B. Bright, D. L. Allara, and C. E. D. Chidsey, "Spontaneously organized molecular assemblies. 4. Structural characterization of $n$-alkyl thiol monolayers on gold by optical ellipsometry, infrared spectroscopy, and electrochemistry," Journal of the American Chemical Society, vol. 109, no. 12, pp. 3559-3568, 1987. 
[20] R. G. Nuzzo, L. H. Dubois, and D. L. Allara, "Fundamental studies of microscopic wetting on organic surfaces. 1. Formation and structural characterization of a self-consistent series of polyfunctional organic monolayers," Journal of the American Chemical Society, vol. 112, no. 2, pp. 558-569, 1990.

[21] M. Ulman, An Introduction to Ultrathin Organic Films, Academic Press, New York, NY, USA, 1991.

[22] D. G. Castner, K. Hinds, and D. W. Grainger, "X-ray photoelectron spectroscopy sulfur $2 p$ study of organic thiol and bisulfide binding interactions with gold surfaces," Langmuir, vol. 12, no. 21, pp. 5083-5086, 1996.

[23] T. Ishida, M. Hara, I. Kojima et al., "High resolution X-ray photoelectron spectroscopy measurements of octadecanethiol self-assembled monolayers on Au(111)," Langmuir, vol. 14, no. 8, pp. 2092-2096, 1998.

[24] T. Ishida, N. Choi, W. Mizutani et al., "High-resolution Xray photoelectron spectra of organosulfur monolayers on $\mathrm{Au}(111): \mathrm{S}(2 \mathrm{p})$ spectral dependence on molecular species," Langmuir, vol. 15, no. 20, pp. 6799-6806, 1999.

[25] F. Buckel, F. Effenberger, C. Yan, A. Gölzhäuser, and M. Grunze, "Influence of aromatic groups incorporated in long-chain alkanethiol self-assembled monolayers on gold," Advanced Materials, vol. 12, no. 12, pp. 901-905, 2000.

[26] C.-J. Zhong, R. C. Brush, J. Anderegg, and M. D. Porter, "Organosulfur monolayers at gold surfaces: reexamination of the case for sulfide adsorption and implications to the formation of monolayers from thiols and disulfides," Langmuir, vol. 15 , no. 2, pp. 518-525, 1999.

[27] J. L. Bahr, E. T. Mickelson, M. J. Bronikowski, R. E. Smalley, and J. M. Tour, "Dissolution of small diameter single-wall carbon nanotubes in organic solvents?" Chemical Communications, no. 2, pp. 193-194, 2001.

[28] J. Liu, A. G. Rinzler, H. Dai et al., "Fullerene pipes," Science, vol. 280, no. 5367, pp. 1253-1256, 1998.

[29] V. Krstic, G. S. Duesberg, J. Muster, M. Burghard, and S. Roth, "Langmuir-Blodgett films of matrix-diluted singlewalled carbon nanotubes," Chemistry of Materials, vol. 10, no. 9, pp. 2338-2340, 1998.

[30] G. S. Duesberg, J. Muster, V. Krstic, M. Burghard, and S. Roth, "Chromatographic size separation of single-wall carbon nanotubes," Applied Physics A, vol. 67, no. 1, pp. 117-119, 1998.

[31] V. V. Zhirnov, E. I. Givargizov, and P. S. Plekhanov, "Field emission from silicon spikes with diamond coatings," Journal of Vacuum Science and Technology B, vol. 13, no. 2, pp. 418421, 1995. 

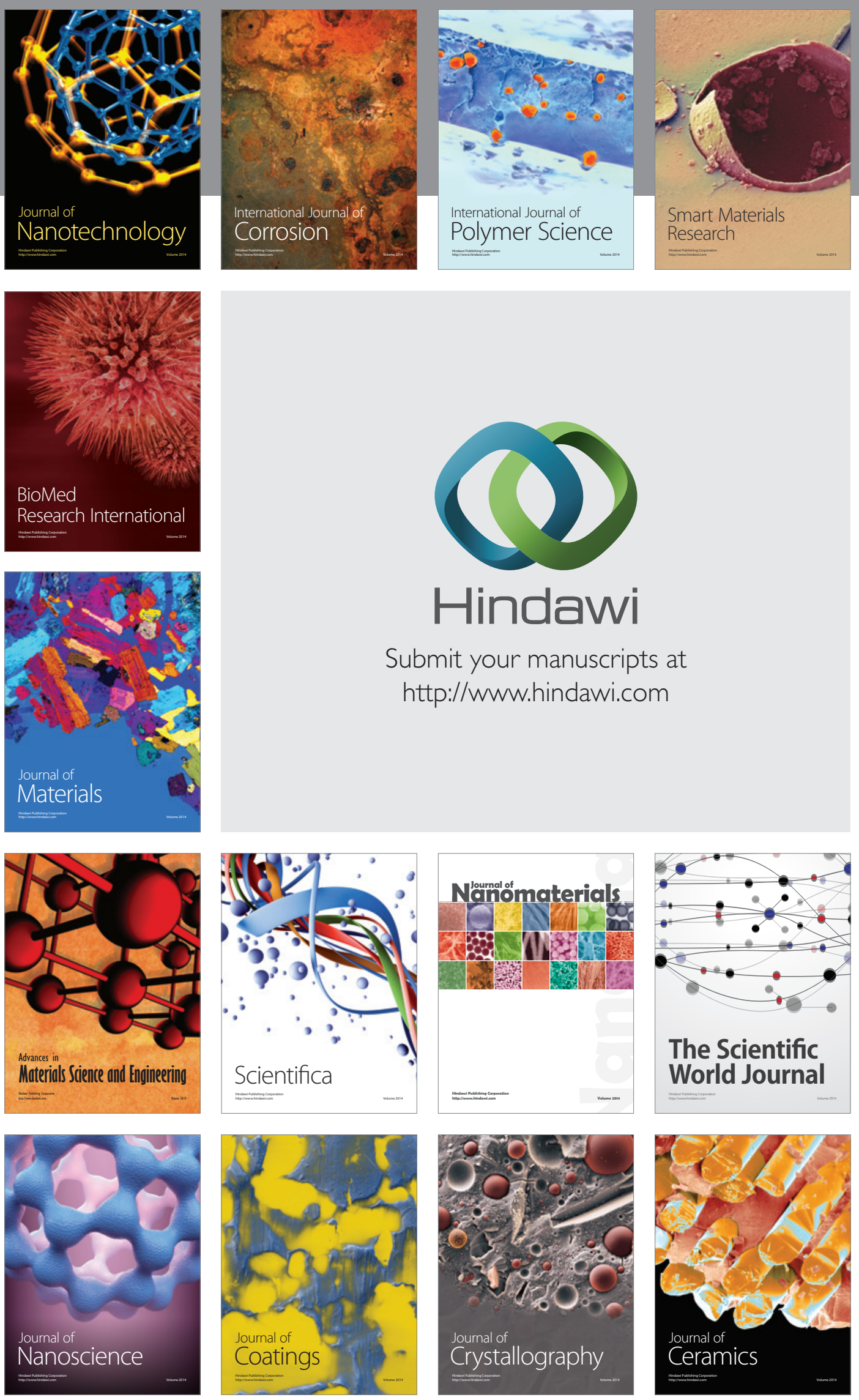

The Scientific World Journal

Submit your manuscripts at

http://www.hindawi.com

\section{World Journal}

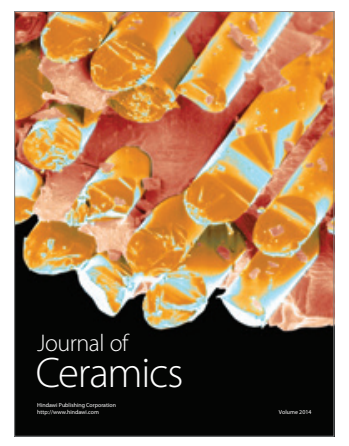

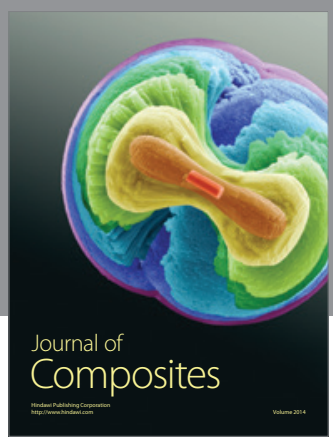
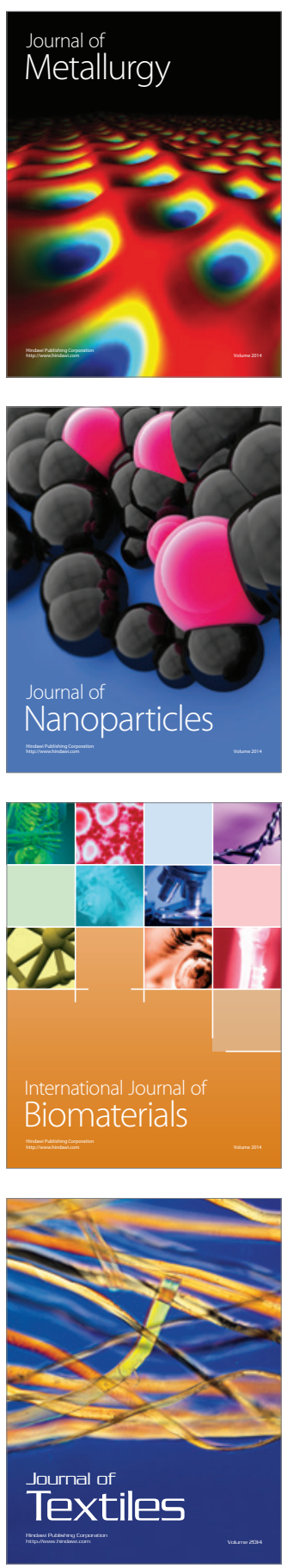